\title{
Sampling and Reconstruction of Surfaces and Higher Dimensional Manifolds
}

Emil Saucan · Eli Appleboim • Yehoshua Y. Zeevi

Published online: 4 June 2008

(C) Springer Science+Business Media, LLC 2008

\section{Erratum to: J Math Imaging Vis}

DOI 10.1007/s10851-007-0048-z

Figures 6 and 7 in the above-referenced article did not print correctly. Please see below for the correct images.
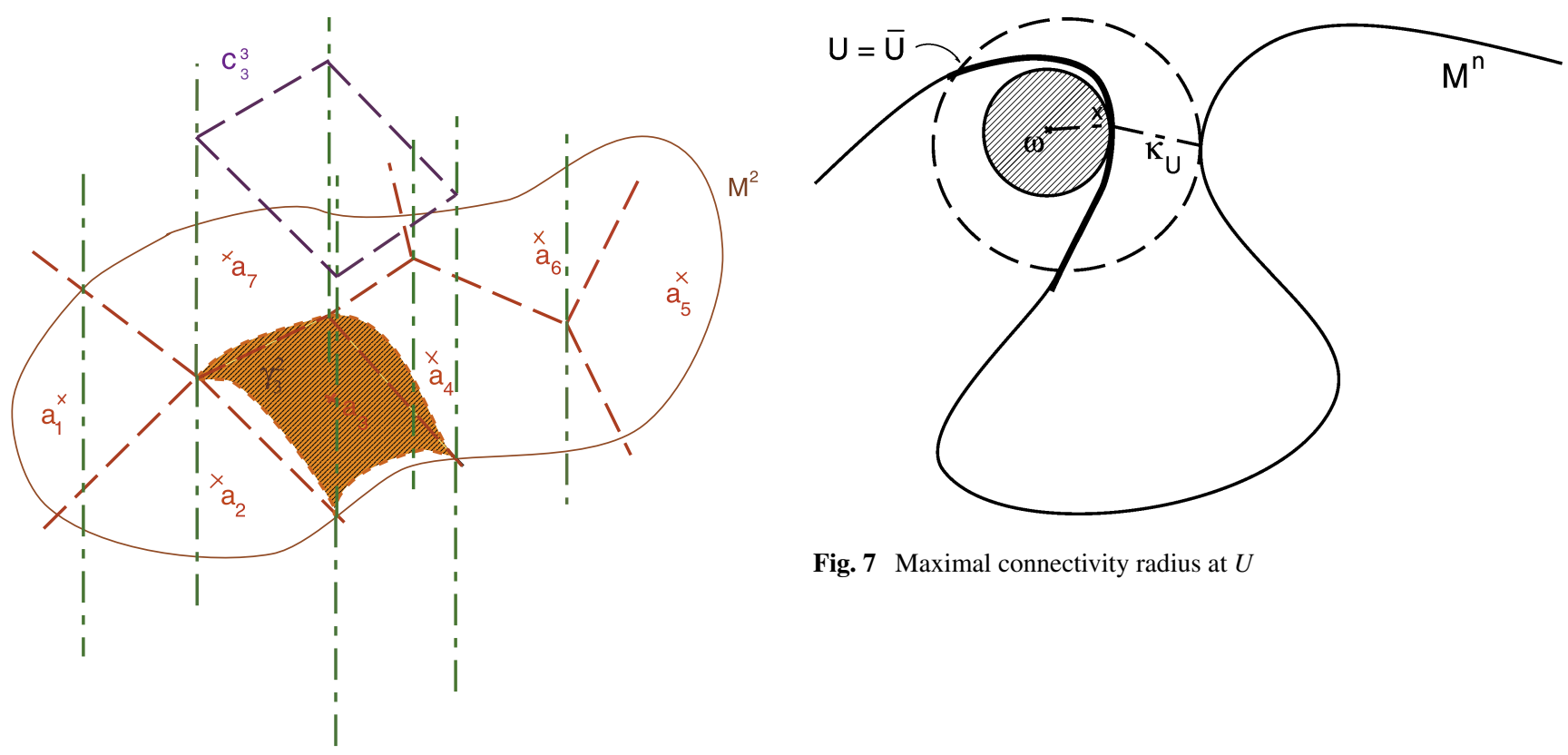

Fig. 7 Maximal connectivity radius at $U$

Fig. 6 Dirichlet (Voronoi) cells—-the compact surface case

The online version of the original article can be found under http://www.dx.doi.org/10.1007/s10851-007-0048-z

E. Saucan $(\varangle) \cdot$ E. Appleboim · Y.Y. Zeevi

Department of Electrical Engineering, Technion, Technion City,

Haifa 32000, Israel

e-mail: semil@ee.technion.ac.il 